\title{
« Sans dieu ni maître » : brouillage des frontières ontologiques, temporelles et mythiques dans L'ogre de Grand Remous de Robert Lalonde
}

\author{
VIVIANE BROCHOT \\ Université Dalhousie
}

Résumé

Cet article se concentre sur les techniques qui ont été utilisées par Robert Lalonde pour repousser les limites des frontières ontologiques et temporelles. C'est grâce à cette destruction minutieuse des frontières que l'auteur remet en question non seulement le mythe dans lequel vivent les héros du roman, mais aussi celui de l'American dream à la québécoise.

\begin{abstract}
Manifest Destiny! They mapped it out, they made pictures of it, they digested it into the great American profit machine. Those were the last bits of the continent, the blank spaces no one had explored. Now here it was, all laid out on a pretty piece of canvas for everyone to see. The golden spike, driven right through our hearts! (Auster, 149)
\end{abstract}

Né à Oka, au Québec, en 1947, Robert Lalonde appartient à la vague d'auteurs québécoisii, qui, en 1980, ont reconnu l'américanité du Québec et ont permis à la littérature québécoise d'entrer pleinement dans la période moderne. Cette ouverture avait été amorcée par Jacques Ferron, le médecin, romancier et politicien de Louiseville, qui avait pris en considération l'altérité anglaise. Il y a donc la vision ferronienne de l'autre, de I'Anglais et des relations qu'entretient le Québec avec l'extérieur. Dans son dernier texte, « Les deux lys », Ferron se montre beaucoup plus pessimiste que ne le fait Lalonde dans son roman. Dans ce texte, Ferron abandonne ses espoirs passés de voir un Québec souverain : « Aurais-je vécu inutilement dans l'obsession d'un pays perdu ? Alors Seigneur, je te le dis : que le Diable m'emporte » (222). Le Québec n'est-il alors qu'un îlot français perdu dans un continent anglophone ? La vision de Lalonde est beaucoup plus globale et américaine, même si, comme nous allons le voir, son Québec américain est résolument contemporain et que l'auteur tente de se débarrasser de l'image d'Epinal selon laquelle il est un paradis perdu sauvage et mythique.

L'ogre de Grand Remous raconte I'histoire de la famille Messier. Des parents, Carmen et Georges, qui disparaissent une nuit au volant d'une Chevrolet bleue pour découvrir le monde en laissant 26000 dollars à leurs enfants à la banque. Charles, Serge et Aline, les trois aînés, tentent de les retrouver et de comprendre leur fuite. Julien, le petit dernier, refuse I'obsession de ses frères et sœurs, se replie dans un monde fictif et sombre dans la folie. Lalonde, à travers les différentes parties de ce roman-quête, raconte l'histoire de la famille Messier du point de vue de chaque enfant, jamais le même, et amène le lecteur à découvrir ce qui s'est vraiment passé cette nuit-là. Dans L'ogre de Grand Remous, Robert Lalonde remet en question et joue avec cette notion de frontière grâce à une version moderne du Petit Poucet de Charles Perrault. Dans ce roman, un réalisateur part à la recherche de ses origines et tente de recomposer le puzzle d'une enfance mythique et sauvage qui a été vécue « sans dieu ni maître ». Lire L'ogre de Grand Remous de Robert Lalonde est une expérience étonnante et déroutante, tant le texte suit des tours et des détours. Tel un labyrinthe, le roman compose un réseau complexe de pistes qui sont autant de voies qui mènent le lecteur à découvrir la vérité. 
L'auteur confronte deux visions antagoniques du lieu qui a bercé l'enfance des protagonistes de son roman : le Grand Remous contemporain que Charles essaie de redécouvrir et le Grand Remous éternel que Julien tente de préserver. C'est à travers la tension entre les différentes frontières qui composent le roman qu'émergera la vérité et que les personnages pourront enfin se libérer du mythe « grand remousien ».

Examinons tout d'abord le texte en lui-même et tout particulièrement les frontières génériques et ontologiques que Lalonde fait voler en éclats. L'auteur a fait appel à de nombreux genres pour composer son roman, participant ainsi à ce que Bakhtine appelle « la carnavalisation de la littérature » (161). Ce roman s'ouvre sur un petit scénario :

Ils remontent dans la Chevrolet, restent encore un peu sans bouger, collés l'un à l'autre. Lui, il pleure pendant qu'elle embrasse ses cheveux mouillés en répétant :

- Mon amour, mon amour, t'es un champion!

Un énorme coup de tonnerre fracasse le ciel, ébranle la pinède, secoue le barrage. Un grondement de fin du monde encore au-dessus de la falaise, plus fort que les chutes... (10)

On peut ensuite lire une lettre que le producteur de Charles lui envoie (on retrouve plus tard le genre épistolaire à plusieurs reprises avec les lettres que s'envoie la fratrie), un journal intime dans lequel Aline consigne les rêves de ses frères et un acte notarié grâce auquel les enfants apprennent qu'ils sont les seuls survivants de leur famille (138-140). On trouve aussi de longs passages du conte de Perrault « Le Petit Poucet » et enfin un télégramme (171). Le manque d'unité générique de ce roman ne signifie pas pour autant que toute notion d'ordre et de structure soit rejetée. C'est à travers les différentes parties que les enfants peuvent exposer leur point de vue sur leur drame. Voici une liste des parties qui composent le roman :

p. 9-10: Scène de départ écrite sous forme d'un petit scénario qui lance l'intrigue. On y apprend qu'un couple veut abandonner ses enfants.

p. 11-13 : Lettre d'André Lapointe, producteur de Charles, réalisateur: constat d'échec sur la capacité de Charles de reconstruire I'histoire des «Quatre orphelins de Grand Remous», sa propre histoire.

p. 17-80 : «Julien le magnifique» (Charles)

p. 83- 149 : «Le vieux divan de la mémoire» (Aline)

p. 153-172 : «Un hiver sur la plage» (Des nouvelles de Serge)

p. 175-182: «Grand Remous» (Julien)

p. 185-189 : «Le barrage» (Aline)

Le roman débute par un petit scénario dans lequel un couple se dispute. La femme veut partir et abandonner ses enfants. Personne ne sait ce qui leur arrive. Le chapitre suivant est une lettre qu'un producteur adresse à un de ses réalisateurs : cette lettre est un constat d'échec. Le réalisateur, Charles, n'a pas réussi à percer le mystère de Grand Remous et de la disparition de ses parents, de « quatre destins qui se croisent sans jamais se rencontrer » (12) Le lecteur, de par sa position privilégiée, a accès à ces quatre destins.

Lire un roman contemporain, c'est aussi combler les vides. Umberto Eco souligne cette lecture active dans Lector in Fabula dans la mesure où « un texte veut qu'on l'aide à fonctionner » (64). Dans $S / Z$, Roland Barthes parle aussi « du lecteur, non plus un consommateur, mais un producteur même du texte » (10). Il revient donc au lecteur de rassembler les différentes pièces qui composent l'intrigue afin de donner un sens au chaos organisé qu'est le roman de Lalonde et ainsi éviter la désagrégation ultime du texte. Ce chaos est annoncé dans la première scène du roman dans laquelle on entend : " Un grondement de fin du monde qui résonne encore au-dessus de la falaise, plus fort que les chutes...» (10). Ajoutons que l'épigraphe placée en 
exergue du texte ne se réduit pas à une fonction décorative mais en est bien la fondation. Elle place, en effet, le lecteur dans une attitude herméneutique, en quête du sens de la citation. Selon Antoine Compagnon dans La Seconde Main ou le travail de la citation, l'épigraphe instaure déjà une attente chez le lecteur : « L'auteur abat ses cartes. Solitaire au milieu d'une page, l'épigraphe représente le livre - elle se donne pour son sens, parfois son contresens -, elle l'induit, elle le résume » (337). Lalonde a choisi de placer en exergue des citations de Deux Cavaliers de l'orage de Jean Giono et du Petit Poucet de Charles Perrault. Le Petit Poucet est un choix assez évident tant les intertextes entre le conte de Perrault et celui de Lalonde sont nombreux. La citation de Giono « Tout est employé à boucher le trou par où coule le sang des tiens » offre aux lecteurs des indices interprétatifs précieux pour la lecture future du roman. L'expression « boucher les trous » peut être interprétée de deux manières : on pourrait la comprendre tout d'abord comme le synonyme du verbe cacher, Julien tentant de dissimuler la vérité aux lecteurs, ainsi qu'à ses frères et à sa sœur. On pourrait aussi considérer cette expression comme l'équivalent du geste de Charles qui tente de combler les vides. Dès le début du texte, le lecteur sait qu'il y a un secret à découvrir et c'est grâce à sa lecture du texte qu'il assemblera toutes les pièces du puzzle. Le texte pourra ainsi trouver une cohérence. Le lecteur réussit là où Charles a échoué en faisant son documentaire « Julien le magnifique » qui était une tentative de comprendre le drame familial que lui et sa famille ont vécu :

Mon cher Charles,

J'ai bien regardé ce premier assemblage de ton Julien le magnifique, qui m'a laissé à tout le moins perplexe. Certes, les images sont belles. Ce Grand Remous, dont tu m'avais tant parlé, apparaît presque aussi mythique que dans tes descriptions, et ton frère est bien évidemment ce personnage étonnant que tu m'avait brossé, un soir de l'an dernier, au bar du studio. Alors, qu'est-ce qui ne va pas ? Eh bien, justement, c'est que ton film ressemble beaucoup trop à ce que tu voulais en faire! Pas une séquence qui ne soit, en tout point, conforme à ce scénario, dont nous avions longuement discuté, et se voulait « un canevas de départ » à partir duquel tu « ouvrirais ailleurs »... (11)

L'échec de Charles, c'est de n'avoir considéré qu'un seul point de vue, le sien, et son échec servira de point de départ à l'enquête que mèneront les lecteurs pour tenter de découvrir la vérité sur le drame qu'ont vécu les personnages. Dans ce roman, le lecteur a le privilège d'avoir accès à toutes les pièces qui composent l'intrigue, le dénouement n'est donc pas une surprise pour lui. L'hybridité, les principes de la fragmentation et de I'indétermination viennent perturber la narration et surtout les narrateurs du roman. Chez Lalonde, comme chez de nombreux auteurs contemporains, l'auteur met en scène des narrateurs hésitants qui ont perdu I'omniscience et la confiance dont bénéficiaient leurs ancêtres. Donner du sens au texte devient donc une tâche beaucoup plus difficile qu'il n'y paraît dans le roman de Lalonde, tant les frontières entre réalité et fiction, entre folie et raison sont ténues.

Le brouillage des frontières est encore accentué par un mélange entre le fictionnel et le réel dans lequel Julien, ses frères et ses sœurs perdent leurs repères. Dans le roman de Lalonde les références au monde des contes de fées, de la littérature et du cinéma abondent : Carmen est comparée à une actrice (24), Julien est comparé par son père à Robinson Crusoe et à Davy Crockett (24) Aline et Charles se « [tiennent] la main comme Hansel et Gretel » (49), Irène, la compagne de Julien est pour ce dernier « La belle au bois dormant » (37). L'obsession de Julien pour Le Petit Poucet est telle que pour lui la fiction se confond avec la réalité. Ainsi, 
quand sa sœur Aline lui raconte Le Petit Poucet et La belle au bois dormant le soir pour s'endormir, le petit Julien dit :

La maison dans la forêt où la reine ogresse fait enfermer ses petits enfants, c'est bien la maison de l'ogre, la même maison, la cabane du géant Trinité ?

Peut-être..

Le prince de La belle au bois dormant, c'est Poucet devenu grand, hein ?

Ça se peut Julien! Laisse-moi continuer!

Les deux histoires sont une seule et même histoire, hein ?

Si tu veux !

C'est une histoire vraie, hein, Aline ? Tu me jures que c'est une histoire vraie ? (130)

L’hésitation entre la fiction et la réalité dans le roman est encore accentuée par la passion de Carmen, la mère, pour Gone With the Wind. Tout au long du roman, elle est systématiquement liée au personnage de Scarlett O’Hara, au point de ne plus faire qu'un avec le personnage de Margaret Mitchell. Au début du roman, elle lit « Autant en emporte le vent, dans son lit à baldaquin fabriqué avec la moustiquaire trouvée dans la grange » (24). Plus loin, Aline raconte :

Carmen prétendait que nos cheveux, que nous ne lavions jamais, nous protégeaient des ardents rayons. Et puis, ça nous faisait des mèches plus pâles, presque blondes, de belles têtes d'Irlandais, petits Ashley de son fameux Gone with the wind. (119)

La comparaison ne s'arrête pas là, Serge évoque la confusion entre les deux femmes : « Il me semble que nous avons pas mal tout essayé, pris tous les trains et tous les avions, relu vingt fois Gone with the wind jusqu'à confondre Carmen et Scarlett » (158).

Penchons-nous maintenant sur les frontières temporelles et analysons le combat entre contemporanéité et éternité que se livrent Charles et Julien. Dans la première partie du roman, « Julien le magnifique », il y a une alternance dans la narration. Julien et Charles tentent tour à tour d'imposer leur voix en prenant en charge la narration d'un chapitre sur deux. Pour accentuer encore l'opposition entre les deux frères, la différence entre les deux points de vue, les chapitres narrés par Julien sont entièrement en italique. Le documentaire de Charles a pour but de comprendre son enfance et ses origines, mais cette tentative de reconstitution est combattue avec force par Julien qui verbalise son désir le plus cher en disant :

Vous avez abandonné votre maudite manie des origines, et de la suite du monde. Vous êtes, tout à coup, avec moi, dans le même temps et le même espace que moi, que la maison, que le vent, que la rivière qu'on devine, cascadant, sans commencement ni fin, par la fenêtre du salon. C'est une chance, notre chance, miraculeuse: nous n'avons personne qui sache, qui dicte, qui empêche. (32)

Dans ce combat entre éternité et contemporanéité, L'ogre de Grand Remous se présente comme la chronique d'un désastre annoncé. Le texte revient systématiquement vers son point d'origine, c'est-à-dire vers la petite scène de départ dans laquelle le couple Messier tente de prendre la fuite, en suivant les souvenirs des enfants, leur correspondance, leurs rencontres à travers les différentes couches temporelles qui composent le roman. Ce télescopage de temporalité semble être le premier signe du désastre dont le propre est d'être, selon Maurice Blanchot, tout à la fois révolu mais toujours imminent : « Nous sommes au bord du désastre sans que nous puissions le situer dans l'avenir : il est plutôt déjà passé, et pourtant nous sommes au bord, ou sous la menace » (7). Sur un plan stylistique, le désastre dont parle Blanchot se manifeste avec des analepses et des prolepses, technique dont Lalonde use et abuse dans ce roman et qui nous rappelle étrangement le geste de 
Charles Messier, le réalisateur qui fait « avancer et reculer la bande sur la table de montage » (19). Le jardin d'Eden dans lequel vivent les enfants, « trois Adam et une Eve » (20), n'échappe pas à la Chute :

Julien qui ne souffrait pas. Julien qui était au paradis. Julien qui ne s'interrogerait jamais, qui ne partirait jamais de Grand Remous, où il était né par miracle, seul et détraqué, peut-être mais non pas abandonné. Le monde lui parlait, lui révélait ses mystères en silence, en silence. Nous, nous étions opaques, raisonneurs, malheureux. Nous ne sommes pas devenus fous. Nous sommes partis. Nous avons quitté la maison, la colline, la rivière. Nous avons déserté le paradis : C'est nous qui nous sommes perdus. (21)

Ce paradis perdu dans lequel les enfants vivent nous rappelle bien sûr le mythe de la frontière américaine ${ }^{\mathrm{iii}}$. Jacques Cabau dans La prairie perdue rappelle que ce mythe va plus loin que celui d'une terre aux ressources inépuisables que tout le monde peut conquérir et exploiter, elle est beaucoup plus qu'un paradis social. C'est en plus

[I]a terre vierge de l'innocence et du paradis perdu, I'image de la civilisation pastorale idyllique par opposition à l'univers urbain. C'est la caution de pureté qui lave l'Amérique de ses péchés, du capitalisme, de l'industrialisation, de ses villes inhumaines. C'est la terre promise, où l'absence de clôture et l'immensité même proclament la bonté, la générosité et la liberté des grands hommes blancs. C'est le terme du voyage de Bougainville, où l'utopie se réalise, où I'homme blanc devient le « bon sauvage » dont rêvait le Siècle des Lumières. (19)

Lalonde, dans son roman, s'applique page après page à déconstruire le mythe de Grand Remous en ternissant son image de paradis sauvage et éternel. Tout en affirmant I'américanité du Québec, il s'évertue petit à petit à déconstruire I'«American dream », pour affirmer la faillite des pères - des parents biologiques et des pères fondateurs - et ainsi abolir la mythique frontière. Notons que Lalonde a exploré ce mythe dans un autre de ses romans, Sept lacs plus au nord, paru en 1993. Dans son article « Une identité frontalière. Altérité et désir métis chez Robert Lalonde et Louis Hamelin », Emmanuelle Tremblay remarque d'ailleurs que le romancier utilise dans le roman un schéma très manichéen dans lequel I'homme blanc s'oppose au sauvage, à I'Indien :

Alors que Michel, le protagoniste, assiste au drame télévisé de l'actualité, c'est une image d'affrontement entre le bien et le mal qui s'impose : « [un] soldat blond, héroïque, propre, debout devant son ennemi noir, le Mohawk, le warrior sanguinaire ». Outre la réactualisation de l'Indien barbare et satanisé, la mythologie du Far West est ici plaquée sur le réel pour en faire une fiction basée sur la lutte sans nuances entre cow-boys et sauvages. (110)

Ce manichéisme dont nous parle Tremblay se double dans le roman d'un désir d'indentification de Michel avec I'Indien qui représente, par toute l'imagerie qu'il véhicule, « le paradis perdu de l'origine autochtone » (111).

La méticuleuse entreprise déconstructioniste de Lalonde se concrétise tout d'abord à l'aide des allersretours incessants entre le présent et le passé. Après la disparition des parents, les enfants tentent de les faire revivre en leur imaginant une vie fictive. C'est à l'aide de cartes, de mappemondes et de livres de géographie que les enfants peuvent créer la vie rêvée de leurs parents :

Aline et moi transportions les livres et les cartes, Serge, les fruits, les cannes à pêches et le filet. L'aventure recommençait, le pique-nique à Veracruz ou aux Antilles. Nous nous laissions prendre au jeu, nous nous instruisions, nous nous préparions à partir, nous aussi sans le savoir. La légende des cartes devenait leur légende à eux, puis, tranquillement, la nôtre. Le point le plus élevé au dessus de la mer : le mont Everest, 8447 mètres. Le plus haut volcan en activité : le Cotopaxi, Equateur, 5896 mètres. Le lac le plus profond : le lac Baïkal, 1742 mètres. L'ile la plus grande : le Groenland, 2175600 kilomètres carrés. (45)

La confrontation entre la vie rêvée des parents et la réalité qu'ils découvrent lorsqu'ils grandissent y est évidente. La vie rêvée des parents est très loin de la réalité que les enfants découvrent : 
Dans un cahier d'école avec un chien colley sur la couverture, Aline avait collé des photographies de la Virginie, découpées dans le National Geographic Magazine. Un champ de tabac au lever du soleil, trois ouvriers noirs («ce sont des esclaves! ») appuyés sur une haute clôture de bois, dans un bas quartier d'Atlanta, un chalutier rempli de coton, comme un char allégorique, dans le port de Savannah. Les légendes sous les photos parlaient d'un pays magnifique, mais perdu, désillusionné. Au milieu d'un cahier, la célèbre photo du coucher de soleil en flammes avec Clark Gable et Vivien Leigh, en silhouettes sombres, découpée dans la jaquette du Gone with the wind de Carmen. Aline avait recopié certains passages du roman, soulignés par notre mère et susceptibles de nous mettre sur une piste. (39)

Dix ans plus tard, je descendais du train, à Atlanta. Je faisais la tournée des hôtels et des motels, avec leurs photos. Il n'y avait plus de clôtures de bois, d'ouvriers noirs en salopette qui souriaient tristement et, surtout, il n'y avait pas, il n'y avait sans doute jamais eu de Georges et de Carmen Messier dans cette ville agitée, moderne, méconnaissable. (40)

Le monde moderne dans lequel les enfants Messier évoluent n'est plus un monde sauvage, mythique, et la quête d'Aline, Serge et Charles est un échec, ils n'ont pas réussi à retrouver leurs parents. C'est en retournant au paradis, à Grand Remous, qu'ils tenteront de percer le mystère de la disparition de leurs parents. Mais la tentative de retour à Grand Remous, au paradis de I'enfance s'avère aussi un échec. Serge, Aline et Charles doivent en effet faire face à la double transgression de Julien, le Petit Poucet. Une erreur de scénario leur permet de découvrir la vérité, le secret de Julien : ils n'ont pas suivi la piste qu'avait préparée leur frère :

Et puis Donald est remonté, il n'a rien dit, mais j'ai compris à son visage, à scruter les mouvements étranges qu'il faisait pour se réchauffer sur l'écluse, qu'il allait dire quelque chose qu'il ne voulait pas dire, mais qu'il devait nous dire, quelque chose qui changerait tout, pour nous. Et, ce qu'il a dit, c'est :

- There is a car, down there! A rusted pale blue Chevrolet!

Serge a poussé un petit cri d'oiseau. Charles et moi nous regardions le courant, sans bouger. C'est alors que j'ai aperçu Julien. Il était sur le seuil de l'ancienne cabane du géant Trinité Lauzon. Une femme se tenait près de lui, un bébé enveloppé de langes dans ses bras. [...] Quand je suis arrivé près de lui, je l'ai pris dans les bras. Il pleurait et répétait :

- Vous avez pas trouvé mes cailloux... Vous avez pas trouvé mes cailloux...

La première transgression est textuelle et symbolique, Julien, le Petit Poucet, est devenu père. Il a fait un enfant à Irène, sa Belle au bois dormant. Il ne peut donc plus prétendre être ce symbole enfantin. La deuxième transgression est beaucoup plus grave, Julien avoue enfin le meurtre des parents dont il a fait basculé la voiture « d'un bon coup d'épaule ». (188) La double transgression de Julien pervertit à jamais Grand Remous, le paradis des enfants Messier, mais en même temps leur permet de résoudre le mystère de leur enfance. Les notions d'éternité et de pureté n'ont plus lieu d'être dans ce lieu que Julien voulait être une forteresse de I'enfance ${ }^{\mathrm{iv}}$.

Écrire consiste à reproduire, à refaire la même chose différemment. Le roman de Lalonde en est un bon exemple, car le texte raconte sans cesse la même histoire. Tout d'abord celle du Petit Poucet, celle des enfants Messier et de Grand Remous, et enfin I'histoire du Québec et de I'Amérique du Nord. Toutes ces histoires sont des variations sur le même thème : la perte et plus particulièrement la perte des origines. 


\section{Références}

Auster, Paul. Moon Palace. London: Penguin books, 1989.

Blanchot, Maurice. L'écriture du désastre. Paris : Gallimard, 1980.

Bakhtine, Michael. La poétique de Dostoïevski, $1^{\mathrm{e}}$ édition 1929. Paris : Les éditions du Seuil, 1970.

Barthes, Roland. S/Z. Paris : Les éditions du Seuil, 1970.

Cabau, Jacques. La prairie perdue. Paris : Les éditions du Seuil, 1966.

Compagnon, Antoine. La Seconde main ou le travail de la citation. Paris : Seuil, 1979.

Eco, Umberto. Lector in Fabula. Le rôle du lecteur ou la coopération interprétative dans les textes narratifs, $1^{\mathrm{e}}$ édition 1979. Paris, Le livre de poche, Biblio Essais, 1985.

Ferron, Jacques. La conférence inachevée : Le pas de Gamelin et autres textes. Montréal : VLB éditeur, 1987.

Lalonde, Robert. L'ogre de Grand Remous. Paris : Les éditions du Seuil, 1992.

Le diable en personne. Paris : Points, 2001.

Tremblay, Emmanuelle. «Une identité frontalière. Altérité et désir métis chez Robert Lalonde et Louis Hamelin. » Études françaises 41 (2005) : 107-124.

Turner, Frederick Jackson. The Frontier in American History http://www.gutenberg.org/files/22994/22994h/22994-h.htm

\footnotetext{
' Cet article a d'abord été présenté sous forme de communication dans le cadre du colloque annuel de l'ALCQ, à Vancouver, en juin 2008.

ii On pensera à des auteurs comme Jacques Poulin ou Jean-Yves Soucy. Plus près de nous, la romancière Lise Tremblay a elle aussi exploré l'américanité montréalaise dans La danse juive, roman dans lequel elle propose à ses lecteurs une critique acide de la culture populaire contemporaine québécoise qui se mondialise de plus en plus.

iii Nous faisons référence ici au concept américain de frontière tel que défini par l'historien Frederick Jackson Turner en 1893 dans The Significance of the Frontier in American History dans lequel il dit : «American social development has been continually beginning over again on the frontier. This perennial rebirth, this fluidity of American life, this expansion westward with its new opportunities, its continuous touch with the simplicity of primitive society, furnish the forces dominating American character. The true point of view in the history of this nation is not the Atlantic coast, it is the Great West. Even the slavery struggle, which is made so exclusive an object of attention by writers like Professor von Holst, occupies its important place in American history because of its relation to westward expansion. In this advance, the frontier is the outer edge of the wave - the meeting point between savagery and civilization. Much has been written about the frontier from the point of view of border warfare and the chase, but as a field for the serious study of the economist and the historian it has been neglected. The American frontier is sharply distinguished from the European frontier $-a$ fortified
} 
boundary line running through dense populations. The most significant thing about the American frontier is that it lies at the hither edge of free land. In the census reports it is treated as the margin of that settlement which has a density of two or more to the square mile. The term is an elastic one, and for our purposes does not need sharp definition. We shall consider the whole frontier belt, including the Indian country and the outer margin of the "settled area" of the census reports. » (http://www.gutenberg.org/files/22994/22994-h/22994h.htm)

iv Robert Lalonde a aussi exploré le thème de la magie de l'enfance et de l'évolution des enfants dans Le diable en personne (1993) dans lequel on peut lire : « La vie est un conte de fée qui perd ses pouvoirs magiques lorsque nous grandissons » (85). 